\title{
Vehicle and Mobile Equipment Mechanics, Installers, and Repairers
}

National Cancer Institute

\section{Source}

National Cancer Institute. Vehicle and Mobile Equipment Mechanics, Installers, and

Repairers. NCI Thesaurus. Code C122531.

The group of professions that include aircraft mechanics and service technicians, automotive technicians and repairers, bus and truck mechanics and diesel eng ine specialists, heavy vehicle and mobile equipment service technicians and mechanics, small engine mechanics, bicycle repairers, recreational vehicle service technicians and tire repairers and changers. 